\title{
Laser decoating of DLC films for tribological applications
}

\author{
A. Zivelonghi ${ }^{1,2} \cdot$ L. Giorleo $^{1}$ - M. Gelfi ${ }^{1}$ - E. Ceretti $^{1}$ - G. M. La Vecchia ${ }^{1}$
}

Received: 15 October 2016 / Accepted: 28 April 2017 /Published online: 21 June 2017

(C) Springer-Verlag London 2017

\begin{abstract}
Damaged DLC coatings usually require remanufacturing of the entire coated components starting from an industrial chemical de-coating step. Alternatively, a complete or local coating repair can be considered. To pursue this approach, however, a local coating removal is needed as first operation. In this context, controlled decoating based on laser sources can be a suitable and clean alternative to achieve a prefixed decoating depth with high accuracy. In the present study, we investigated a laser-based decoating process executed on multilayered DLC films for advanced tribological applications (deposited via a hybrid PVD/PE-CVD technique). The results were acquired via multifocal optical digital microscopy (MFODM), which allowed high-resolution 3D surface reconstruction as well as digital profilometry of the lasered and unlasered surface. The study identifies the most critical process parameters which influence the effective decoating depth and the postdecoating surface roughness. In particular, the role of pulse overlap (decomposed along orthogonal directions), laser fluence, number of lasing passes and assist gas is discussed in text. A first experimental campaign was designed to identify the best conditions to obtain full decoating of the DLC + DLC:Cr layers. It was observed that decreasing the marking speed to $200 \mathrm{~mm} / \mathrm{s}$ was necessary to obtain a sufficient pulse overlap and a nearly
\end{abstract}

Electronic supplementary material The online version of this article (doi:10.1007/s00170-017-0482-z) contains supplementary material, which is available to authorized users.

\section{A. Zivelonghi}

alessandro.zivelonghi@unibs.it; zasaconsulting@gmail.com

1 Department of Mechanical and Industrial Engineering, University of Brescia, I-25123 Brescia, Italy

2 Zasa Consulting - Studio di consulenza ingegneristica, I-37029 San Pietro in Cariano, Italy planar ablation profile. By operating with microsecond pulses and $1 \mathrm{~J} / \mathrm{cm}^{2}$ (fairly above the ablation threshold), less than 10 passes were needed to obtain full decoating of the lasered area with an etching rate of $1.1 \mu \mathrm{m} / \mathrm{loop}$. Further experiments were then executed in order to minimise the roughness of the rest surface with the best value found at around $0.2 \mu \mathrm{m}$. Limited oxidation but higher $R_{a}$ values were observed in Ar atmosphere.

Keywords Laser decoating $\cdot$ Laser etching $\cdot$ DLC $\cdot$ PE-CVD

\section{Introduction}

DLC films are employed as protective coatings in different engineering contexts due to excellent material properties like high hardness, chemical stability, optical transparency, dielectricity and biocompatibility $[1,2]$. These coatings also possess a very favourable tribological behaviour as low-friction films for different mechanical components. Just to mention a few examples, superlubricity of DLC films applied to engine components was recently shown by Kano and coworkers [3, 4], whereas excellent fatigue performance for biomedical load bearing joints was demonstrated by Thorwarth et al. in [5]. To author's knowledge, however, very little attention has been devoted so far to the issue of decoating of DLC films deposited via physical or chemical vapour deposition (PVD/CVD) processes.

In spite of being protective, DLC films can still be subjected to various damage processes like mechanical impact, delamination, cracking, wear and corrosion. In [5], stresscorrosion-cracking (SCC) from oxygen contamination was shown to possibly cause massive delamination of DLC coatings. Damaged coatings usually require remanufacturing of the entire coated components from scratch. Alternatively, a complete or local coating repair can be considered. To pursue this approach, however, a local coating removal is needed as 
first operation. In this context, controlled decoating based on laser sources can be a suitable and clean alternative to achieve a pre-fixed decoating depth with high accuracy. Moreover, it allows to control the surface finishing before the second processing stage, i.e. the redeposition process, takes place. Removing a DLC coating via laser ablation may offer some advantages over common removal techniques. Mechanical grinding, for instance, is usually hand-performed, difficult to automate and responsible for residual mechanical stress or damage. On the other hand, chemical routes are suitable to large surfaces but also difficult to control when micrometric or nanometric multilayer structures are involved. In addition, they involve acid solutions and polluting chemicals as in the method patented by Hsiao [6]. Another approach based on plasma jet is worth mentioning [7], although the very low etching rate achievable $(\sim 60 \mathrm{~nm} / \mathrm{min})$ makes this method unsuitable for thick DLC coatings (total thickness $\geq 5-10 \mu \mathrm{m}$ ) typical of tribo-mechanical applications.

A viable alternative is offered by laser ablation/laser etching processes. Upon these processes are based laser cleaning techniques which aim at removing rust, oil and unwanted particles from metallic surfaces [8-10]. Removal of rust and carbonaceous deposits from carbon steel usually require high power densities $\left(>10 \mathrm{MW} / \mathrm{cm}^{2}\right)$ and short pulses $(\leq 10 \mathrm{~ns})[8$, 9]. On the other hand, removal of thick DLC layers can be achieved with longer pulses $(>1 \mu \mathrm{s})$ and correspondingly lower power density $\left(<1 \mathrm{MW} / \mathrm{cm}^{2}\right)[11]$.

So far, laser ablation on DLC films was mainly conceived as a laser surface texturing (LST) technique and not as a cleaning or decoating technique. The work of Amanov et al. [12] and Schum and al. [13], for instance, showed how regular arrays of dimples obtained via LST on DLC films were able to reduce the friction coefficient considerably under oil lubrication. In recent years, LST has become a key technology for enhancing specific tribological properties of DLC coatings [14-17].

In the present work, we are showing controlled laser decoating of DLC films based on planar ablation. In the proposed method, the effective decoating depth and the post-decoating surface roughness were controlled via high-resolution 3D optical digital microscopy (3DODM) in combination with independent calotest measurements. Parameters of the LST process were carefully investigated in order to attain complete removal of the DLC film and, at the same time, planarity and limited oxidation of the rest surface to allow subsequent redeposition.

Nowadays, complex multilayer DLC architectures with several doped layers and metallic adhesion interlayers are encountered in the most advanced tribology applications [18, 19]. During decoating and repair of such multilayered films, controlling the ablation depth with micrometric accuracy becomes a critical requirement, and it is the main subject of the present paper.

\section{Experiment and method}

A low-power (8 W) $Q$-switched laser source (LEP Lee Laser, USA) coupled with galvanometric optics and sample holder with adjustable focal distance was employed as laser decoating setup (Fig. 1a). The laser source $\left(\mathrm{Nd}: \mathrm{YVO}_{4}\right.$, $\lambda=532 \mathrm{~nm}$ ) was operated in pulsed mode with long pulse duration $(10 \mu \mathrm{s})$. To impose a remote control of the pulses along the $x$ and $y$ directions, the outgoing laser beam was collimated in galvanometric mirrors as illustrated in Fig.1b. DLC-coated samples with diameter of $30 \mathrm{~mm}$ each were mounted on a holder at a fixed focal distance from the objective lens $(160 \mathrm{~mm})$. Due to the several configurations tested on each sample, the individual lasing area was limited to $3 \times 3 \mathrm{~mm}^{2}$.

DLC films were growth on ultrasound-cleaned and carefully polished AISI 1040 C40 carbon steel. The films were deposited in a single-batch operation, using a hybrid proprietary PVD-CVD coating system (Powerflex 1100 CR from Protec Surface Technologies [20]). The system is equipped with magnetron sputtering (MS) and cathodic-arc evaporation (CAE) PVD modules as well as with a radio-frequency plasma-enhanced CVD (PE-CVD) source. Process parameters are proprietary. The steel substrate was first treated in ultrademineralised water and then sputter-cleaned with $\operatorname{Ar}(2 \mathrm{~Pa})$ in the evacuated deposition chamber (pressure $5 \times 10^{-3} \mathrm{~Pa}$ ). First, a $0.4-\mu \mathrm{m}$-thick metallic $\mathrm{CrC} / \mathrm{Cr}$ interlayer was deposited via cathodic arc evaporation (CAE-PVD) using solid targets. Then, a doped DLC:Cr layer $(4 \mu \mathrm{m})$ was deposited on top of the interlayer by a continuous hybrid CAE-PVD + PE-CVD process, followed by a top layer of pure DLC $(2.2 \mu \mathrm{m})$ from nanoliquid diamond (NLD) precursors deposited via PECVD. The resulting multilayer structure was first measured with ball calotest (CSM Instruments) to get independent thickness measurements taken as reference (Fig. 2).

Lased samples with different lasing parameters were analysed via multifocal optical digital microscopy (Hirox KH-8700 equipped with a dual-illumination revolver zoom optic). The microscope was operated in mixed mode (co-axial + dark field) to overcome DLC transparency. Multiple scans were necessary to scan a sufficiently extended area per tested configuration (about $1 \times 1 \mathrm{~mm}^{2}$ ) at the required magnification $(\times 1000)$. This was achieved moving the motorised sample table on a $5 \times 5$ position array with intervals of $\sim 200 \mu \mathrm{m}$ along the $x$ and $y$ axes. Multifocal images in tiling mode allowed correct reconstruction in 3D of the lased + unlased surfaces.

\subsection{Laser process parameters}

A description of all experimental parameters is provided in Table 1. In experiments, a number of fixed and varied parameters were involved. The fixed parameters were focal length (FL), laser spot diameter $(D)$, frequency $(f)$ and pulse length 
a

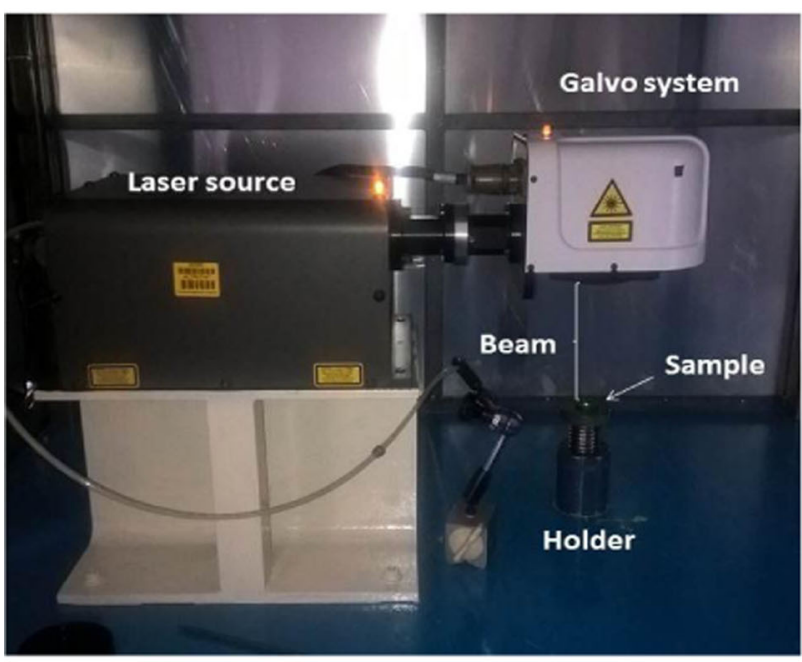

C

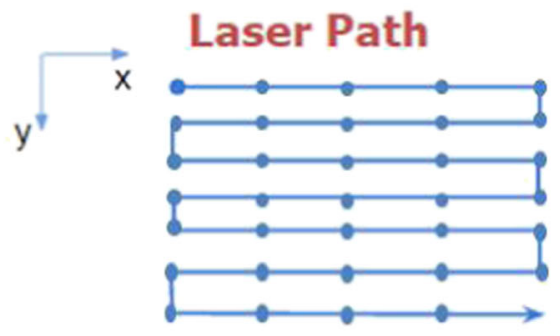

Fig. 1 a Laser decoating experimental setup. b Schematic view of laser decoating on multilayer sample (not in scale). c Laser path and spot centre array (blue points) in the $x-y$ plane. d Distance between pulses in

$(d t)$, whereas the varied controlling factors were laser power $(P)$, beam scanning velocity $(v)$, pulse frequency $(f)$, pulse duration $(d t)$, filling line gap (flg), overlap percentages between consecutive pulses decomposed along orthogonal directions $\left(\mathrm{OL}_{x}\right.$ and $\left.\mathrm{OL}_{y}\right)$ and number of lasing loops $\left(N_{\text {loop }}\right)$. The flg parameter is defined as the distance between two parallel lines of the laser path. The selected laser path was a rectangular serpentine pattern with parallel lines filled from top to bottom and from left to right (Fig.1c). Because of the rectangular lasing pattern, it was possible to decompose the pulse overlap along the two in-plane orthogonal directions. As shown in Fig. 1d, the overlap percentages $\mathrm{OL}_{x}$ and $\mathrm{OL}_{y}$ were indirectly controlled by the flg parameter and the ratio $v / f$, respectively $\left(\mathrm{OL}_{x}=1-\mathrm{flg} / D\right.$ and $\left.\mathrm{OL}_{y}=1-v / f D\right)$.

The values of all varied laser parameters (directly and indirectly controlled) are listed in Table 2. Three levels of lasing speed $\left(v=2500,600,200 \mathrm{~mm} / \mathrm{s}\right.$, corresponding to $\mathrm{OL}_{x}=-25,70$ and $90 \%$ ) and four levels of lasing loops $\left(N_{\text {loop }}=5,10,20\right.$ and 30$)$ were b

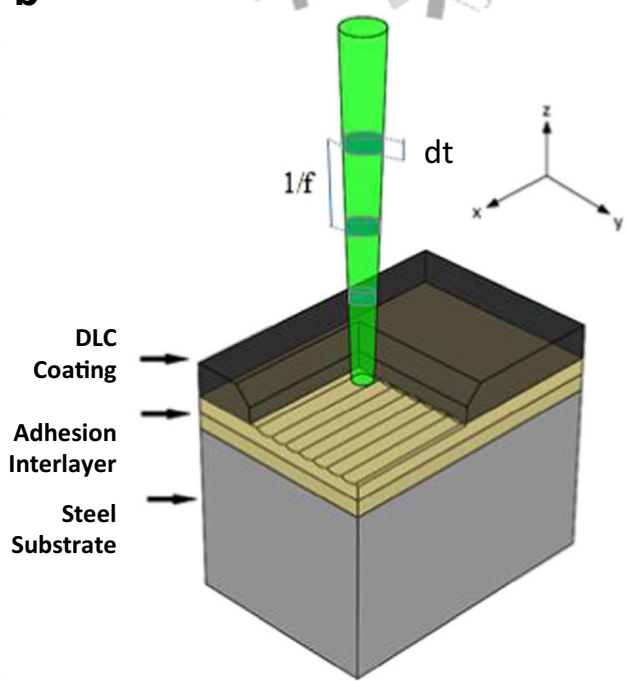

d

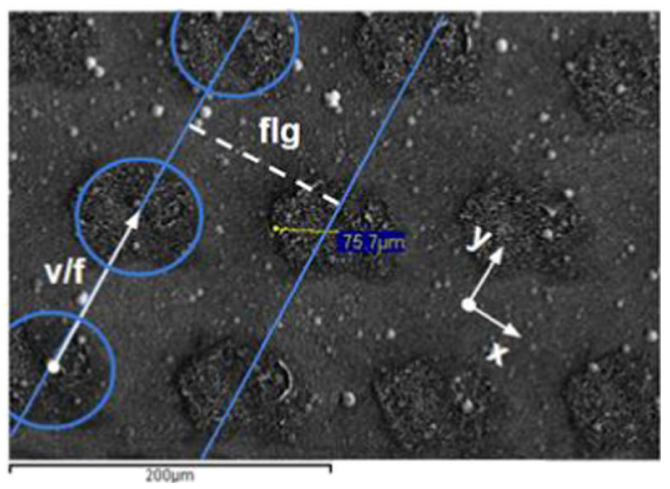

orthogonal directions (v/f and flg) on the DLC surface. The pulses are non-overlapping in this case

explored. The flg parameter was changed over two levels $(0.1$ and $0.01 \mathrm{~mm}$ ), corresponding to 0 and $90 \%$ of $\mathrm{LL}_{y}$ overlap. A preliminary campaign at low fluence performed with non-overlapping pulses $\left(F=0.1 \mathrm{~J} / \mathrm{cm}^{2} ;\right.$ flg $=0.1 \mathrm{~mm} ; v=2500 \mathrm{~mm} / \mathrm{s} ; N_{\text {loop }}=10,20$, 30 ) showed that even at $N_{\text {loop }}=30$, the DLC layer appeared only slightly damaged. For this reason, during all further configurations, the fluence value was increased to $1 \mathrm{~J} / \mathrm{cm}^{2}$ (fairly above the ablation threshold of DLC, which is reported in the interval of 0.2 $\left.0.3 \mathrm{~J} / \mathrm{cm}^{2}[11]\right)$.

\subsection{Ablation depth measurement}

Average and maximum ablation depth were estimated from several different line profiles per sample from surface reconstruction via 3D-ODM, whose total length $L$ was compliant according to DIN EN ISO 4288. Exemplary reconstructions and corresponding profiles are shown in Figs. 2 and 3. Given a discrete line profile $z_{i}\left(x_{i}\right)$ starting outside the lased region, two 
Table 1 Laser setup parameters and material data used in the present study
Laser

Mode

Wavelength

Spot diameter $(D)$

Focusing length (FL)

Frequency $(f)$

Duty cycle $(d c)$

Pulse duration $(d t)$

Peak power

Max power (average)

Max power density

Marking speed ( $v$ )

Number of loops $\left(N_{\text {loop }}\right)$

Filling line gap (flg)

$\mathrm{OL}_{x}=1-\mathrm{flg} / D$

$\mathrm{OL}_{y}=1-v / f D$

Laser path

Lasing atmosphere

Material

Coating type

Substrate

Interlayer

Deposition technique

Lased area per configuration
$Q$-switching pulsed

$532 \mathrm{~nm}$

$0.1 \mathrm{~mm}$

$160 \mathrm{~mm}$

$20 \mathrm{kHz}$

$20 \%$

$10 \mu \mathrm{s}$

$40 \mathrm{~W}$

$8 \mathrm{~W}$

$10 \mathrm{MW} / \mathrm{cm}^{2}$

$200-2500 \mathrm{~mm} / \mathrm{s}$

$1-30$

$0.01-0.1 \mathrm{~mm}$

$0,90 \%$

$-25,70,90 \%$

Serpentine

Air 1 bar/Ar 5 bar

$\mathrm{DLC}+\mathrm{DLC}: \mathrm{Cr}(6.2 \mu \mathrm{m})$

C40 EN 10083

$\mathrm{CrC} / \mathrm{Cr}(0.4 \mu \mathrm{m})$

PE-CVD $(2.2 \mu \mathrm{m}$ DLC $)$

Hybrid CAE-PVD + PE-CVD (4 $\mu \mathrm{m}$ Cr:DLC)

CAE-PVD $(0.2 \mu \mathrm{m} \mathrm{CrC}+0.2 \mu \mathrm{m} \mathrm{Cr})$

$\leq 3 \times 3 \mathrm{~mm}^{2}$
Table 2 Varied laser parameters and related configuration ID

\begin{tabular}{lllllllll}
\hline ID & $P_{\text {avg }}(\mathrm{W})^{\mathrm{a}}$ & $\mathrm{flg}(\mathrm{mm})^{\mathrm{a}}$ & $v(\mathrm{~mm} / \mathrm{s})^{\mathrm{a}}$ & $N^{\mathrm{a}}$ & $F\left(\mathrm{~J} / \mathrm{cm}^{2}\right)$ & $\mathrm{OL}_{x}$ & OL $_{y}$ & Full DLC removal \\
\hline$\# 1$ & 0.65 & 0.1 & 2500 & 1 & 0.1 & $-25 \%$ & $0 \%$ & OFF \\
$\# 2$ & 0.65 & 0.1 & 2500 & 10 & 0.1 & $-25 \%$ & $0 \%$ & OFF \\
$\# 3$ & 0.65 & 0.1 & 2500 & 20 & 0.1 & $-25 \%$ & $0 \%$ & OFF \\
$\# 4$ & 0.65 & 0.1 & 2500 & 30 & 0.1 & $-25 \%$ & $0 \%$ & OFF \\
$\# 5$ & 2.09 & 0.1 & 2500 & 1 & 1 & $-25 \%$ & $0 \%$ & OFF \\
$\# 6$ & 2.09 & 0.1 & 2500 & 10 & 1 & $-25 \%$ & $0 \%$ & OFF \\
$\# 7$ & 2.09 & 0.1 & 2500 & 20 & 1 & $-25 \%$ & $0 \%$ & OFF \\
$\# 8$ & 2.09 & 0.1 & 2500 & 30 & 1 & $-25 \%$ & $0 \%$ & OFF \\
$\# 9$ & 2.09 & 0.01 & 2500 & 5 & 1 & $-25 \%$ & $90 \%$ & OFF \\
$\# 10$ & 2.09 & 0.01 & 2500 & 10 & 1 & $-25 \%$ & $90 \%$ & OFF \\
$\# 11$ & 2.09 & 0.01 & 2500 & 20 & 1 & $-25 \%$ & $90 \%$ & OFF \\
$\# 12$ & 2.09 & 0.01 & 2500 & 30 & 1 & $-25 \%$ & $90 \%$ & OFF \\
$\# 13$ & 2.09 & 0.01 & 600 & 5 & 1 & $70 \%$ & $90 \%$ & OFF \\
$\# 14$ & 2.09 & 0.01 & 600 & 10 & 1 & $70 \%$ & $90 \%$ & OFF \\
$\# 15$ & 2.09 & 0.01 & 600 & 20 & 1 & $70 \%$ & $90 \%$ & OFF \\
$\# 16$ & 2.09 & 0.01 & 600 & 30 & 1 & $70 \%$ & $90 \%$ & OFF \\
$\# 17$ & 2.09 & 0.01 & 200 & 10 & 1 & $90 \%$ & $90 \%$ & ON \\
\hline
\end{tabular}

The ON/OFF output variable refers to complete DLC removal along $z$

${ }^{a}$ Directly controlled parameters 
a

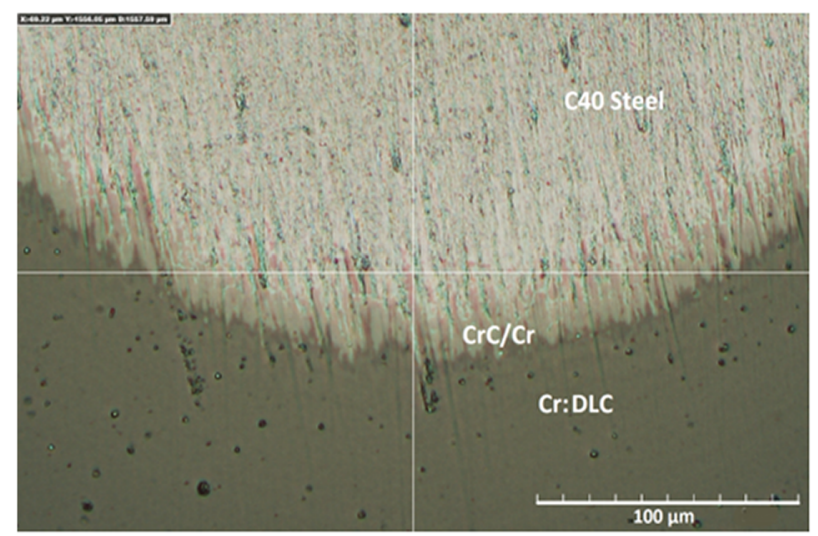

b

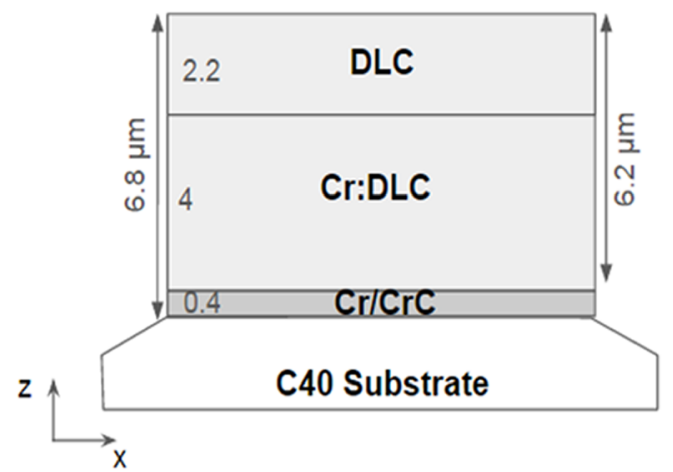

Fig. 2 a Ball imprint from calotest imaged with ODM. The thin $\mathrm{CrC} / \mathrm{Cr}$ interlayer is visible at the interface between DLC and C40 substrate. b Multilayer thicknesses as measured from calotest

estimators of total ablation depth were introduced as delta operators (considering the $x$ axis direction parallel to the lasing speed direction).

The first one, $\Delta z_{\max }$, estimates the maximum ablation depth of a decoating texture by averaging on the five deepest valleys of an ODM profile of total length $L$ along $x$ :

$\Delta z_{\max }=\frac{1}{5} \sum_{j=1}^{5}\left|z_{\min }\left(x_{j}\right)-\bar{z}_{\mathrm{dlc}}\right|$

where $z_{\min }$ contains the local minima of the profile in ascending order and $\bar{z}_{\mathrm{dlc}}$ is the reference height of the DLC coating, measured outside the lased zone. Due to possible local variation of the effective coating thickness, $\bar{z}_{\text {dlc }}$ was recalculated on each profile according to the following formula: $\bar{z}_{\mathrm{dlc}}=$ $\frac{1}{N} \sum_{\forall x_{i}>L_{\mathrm{dlc}}} z_{i}\left(x_{i}\right)$, where $L_{\mathrm{dlc}}$ is the profile ordinate corresponding to the origin of the untouched DLC region and $N$ is the number of profile points considered.

The estimator $\Delta z_{\max }$ is obviously not suitable to check a full decoating condition (complete film removal in all directions) since it cannot distinguish between a channelling texture from a full decoating texture with the same depth (providing the same result in both cases). A second average estimator was therefore introduced, which runs over all profile points of the lased region:

$\Delta z_{\mathrm{avg}}=\frac{1}{N} \sum_{\forall x_{i}<L}\left|z_{i}\left(x_{i}\right)-\bar{z}_{\mathrm{dlc}}\right|$

This estimator outputs an average ablated thickness and can check whether one laser configuration is able to effectively achieve full decoating. In fact, because of symmetry of the laser path (producing parallel lines along the whole lased surface), in order to test full decoating, it is sufficient to test $\Delta z_{\text {avg }}$ along the direction perpendicular to the ablation channels (Fig. 3).

To assess full decoating on the several configurations tested, an ON-OFF criterion was defined comparing decoating thickness values from ODM with independent thickness measurements obtained from calotest, the latter one assumed as reference. Given a DLC layer thickness from calotest $t_{\mathrm{dlc}}^{\text {calo }}$, the used criterion for full decoating simply reads

$\Delta z_{\text {avg }}>t_{\mathrm{dlc}}^{\text {calo }}+\sigma_{\Delta z-\text { avg }}$

where $\sigma_{\Delta z}$ decoating is the standard deviation of $\Delta z_{\text {avg }}$. It is noted that the above inequality expresses a statistically conservative condition since the reference thickness $t_{\mathrm{dlc}}^{\text {calo }}$ is increased by the profile measurement error.

\subsection{Rest surface optimisation}

Selected configurations satisfying Eq. 3 were further analysed considering the post-decoating surface roughness $R_{a}$ as a figure of merit. Hence, additional configurations were introduced at $200 \mathrm{~mm} / \mathrm{s}\left(N_{\text {loop }}=7\right.$ and 15$)$ in order to explore a possible local minimum of $R_{a}$. Finally, the sequence at $200 \mathrm{~mm} / \mathrm{s}$ was entirely repeated in Ar to study oxidation of the rest surface and influence of the assist gas Ar. All configurations investigated in this second optimisation stage are listed in Table 3.

\section{Results}

\subsection{Surface texturing and influence of marking speed}

Ablated surfaces at different lasing speed were first inspected via optical digital microscopy (Fig. 3). In these configurations, the pulse overlap along $y$ was kept fixed to $90 \%$ by fixing the flg parameter to $0.01 \mathrm{~mm}$, whereas the pulse overlap along $x$ was varied from -25 to $90 \%$ by varying accordingly the marking speed. As evident in Fig. 3a, b at high marking speed $(2500 \mathrm{~mm} / \mathrm{s})$, the result is a texture with well-separated and 


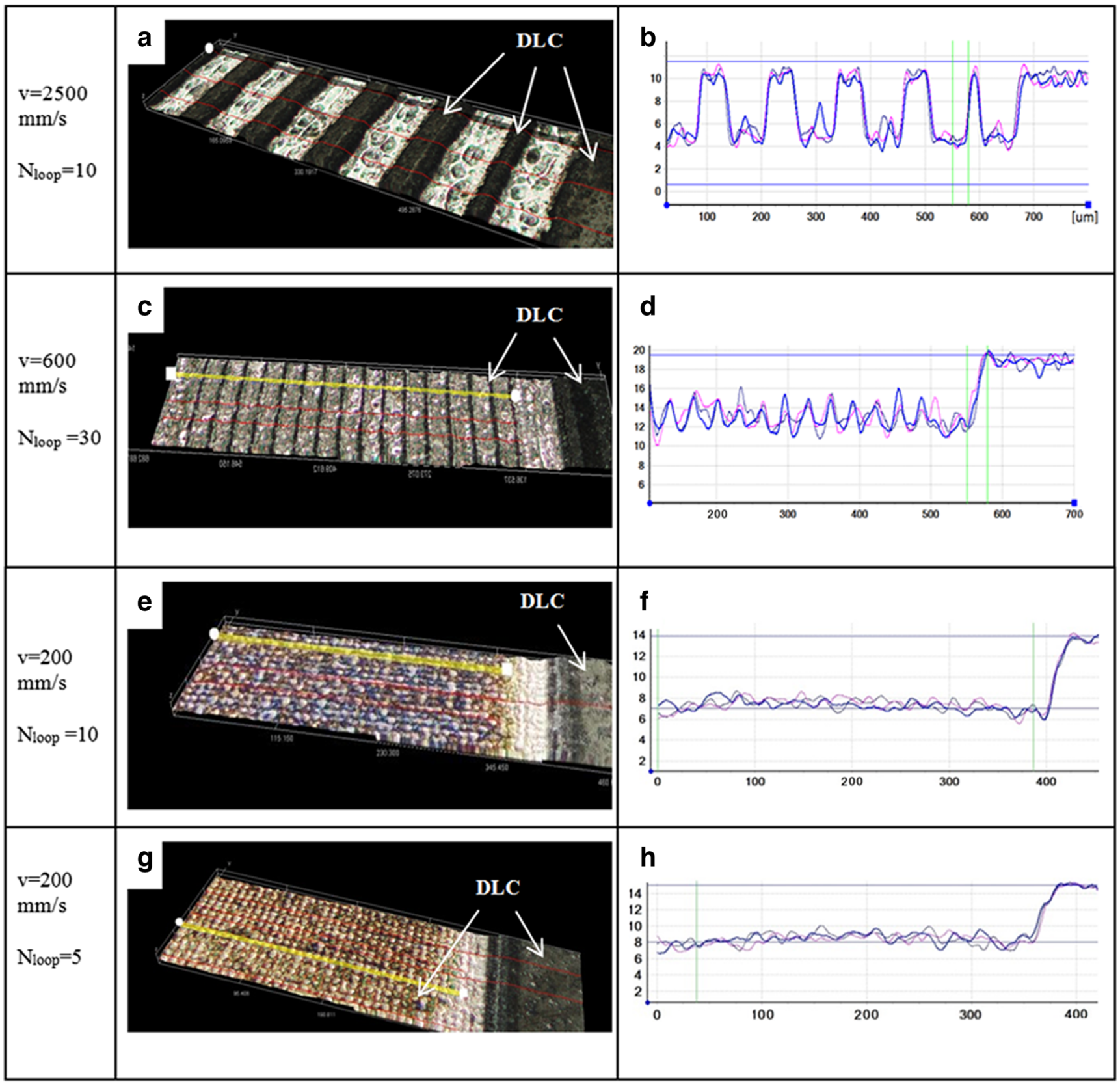

Fig. 3 3D surface reconstructions from optical digital microscopy (Hirox KH- $8700, \times 1000$ magnification, mixed illumination) and corresponding depth profiles

parallel ablation channels within zones of untouched DLC. At this speed, channelling was observed at all $N_{\text {loop }}$ values.

At $600 \mathrm{~mm} / \mathrm{s}$, a slight "channelling" effect is still observable even after 30 loops as confirmed by the thin residual DLC ridges (thickness $<2 \mu \mathrm{m}$ ) visible on the rest surface (Fig. 3c). This fact is rather surprising; given the high theoretical overlap (70\% along $x$ and $90 \%$ along $y$ ) and the high number of passes, one had expected a full decoating texture. However, the inhomogeneous energy distribution within a single laser pulse must be also considered. Since the energy distribution within one pulse is peaked at the spot centre, a lower lasing energy overlap results at the boundary between two parallel ablation channels, eventually leading to fluence values which are locally below the ablation threshold. At $200 \mathrm{~mm} / \mathrm{s}$ (90\% overlap along both $x$ and $y$ ), the resulting total energy distribution over two consecutive pulses lies entirely above the DLC ablation threshold and a planar decoating texture is observed without channelling effect (Fig. 3e, g). However, some DLC rests at the interface with the underlying $\mathrm{CrC}$ layer are still visible after five loops (Fig. 3g).

\subsection{Depth measurements and conditions for full decoating}

Results from 3D-ODM profilometry compared to independent calotest measurements are shown in Fig. 4a, b. Error bars 
Table 3 Parameters for the postdecoating surface optimisation campaign

\begin{tabular}{lllllllll}
\hline ID & $P_{\text {avg }}(\mathrm{W})^{\mathrm{a}}$ & flg $(\mathrm{mm})^{\mathrm{a}}$ & $v(\mathrm{~mm} / \mathrm{s})^{\mathrm{a}}$ & $N^{\mathrm{a}}$ & $F\left(\mathrm{~J} / \mathrm{cm}^{2}\right)$ & $\mathrm{OL}_{x}$ & OL $_{y}$ & Full DLC removal \\
\hline$\# 18$ & 2.09 & 0.01 & 200 & 5 & 1 & $90 \%$ & $90 \%$ & OFF \\
$\# 19$ & 2.09 & 0.01 & 200 & 7 & 1 & $90 \%$ & $90 \%$ & ON \\
$\# 20$ & 2.09 & 0.01 & 200 & 10 & 1 & $90 \%$ & $90 \%$ & ON \\
$\# 21$ & 2.09 & 0.01 & 200 & 15 & 1 & $90 \%$ & $90 \%$ & ON \\
$\# 22$ & 2.09 & 0.01 & 200 & 20 & 1 & $90 \%$ & $90 \%$ & ON \\
$\# 23$ & 2.09 & 0.01 & 200 & 30 & 1 & $90 \%$ & $90 \%$ & ON \\
$\# 18 \mathrm{Ar}$ & 2.09 & 0.01 & 200 & 5 & 1 & $90 \%$ & $90 \%$ & ON \\
$\# 19 \mathrm{Ar}$ & 2.09 & 0.01 & 200 & 7 & 1 & $90 \%$ & $90 \%$ & ON \\
$\# 20 \mathrm{Ar}$ & 2.09 & 0.01 & 200 & 10 & 1 & $90 \%$ & $90 \%$ & ON \\
$\# 21 \mathrm{Ar}$ & 2.09 & 0.01 & 200 & 15 & 1 & $90 \%$ & $90 \%$ & ON \\
$\# 22 \mathrm{Ar}$ & 2.09 & 0.01 & 200 & 20 & 1 & $90 \%$ & $90 \%$ & ON \\
$\# 23 \mathrm{Ar}$ & 2.09 & 0.01 & 200 & 30 & 1 & $90 \%$ & $90 \%$ & ON \\
\hline
\end{tabular}

${ }^{\mathrm{a}}$ Directly controlled parameters of $\pm 0.5 \mu \mathrm{m}$ are due to instrumentation error and layer thickness irregularity during deposition. Green markers refer to engraved channelling texture obtained at high scanning velocity $(2500 \mathrm{~mm} / \mathrm{s})$, whilst black and red markers refer to

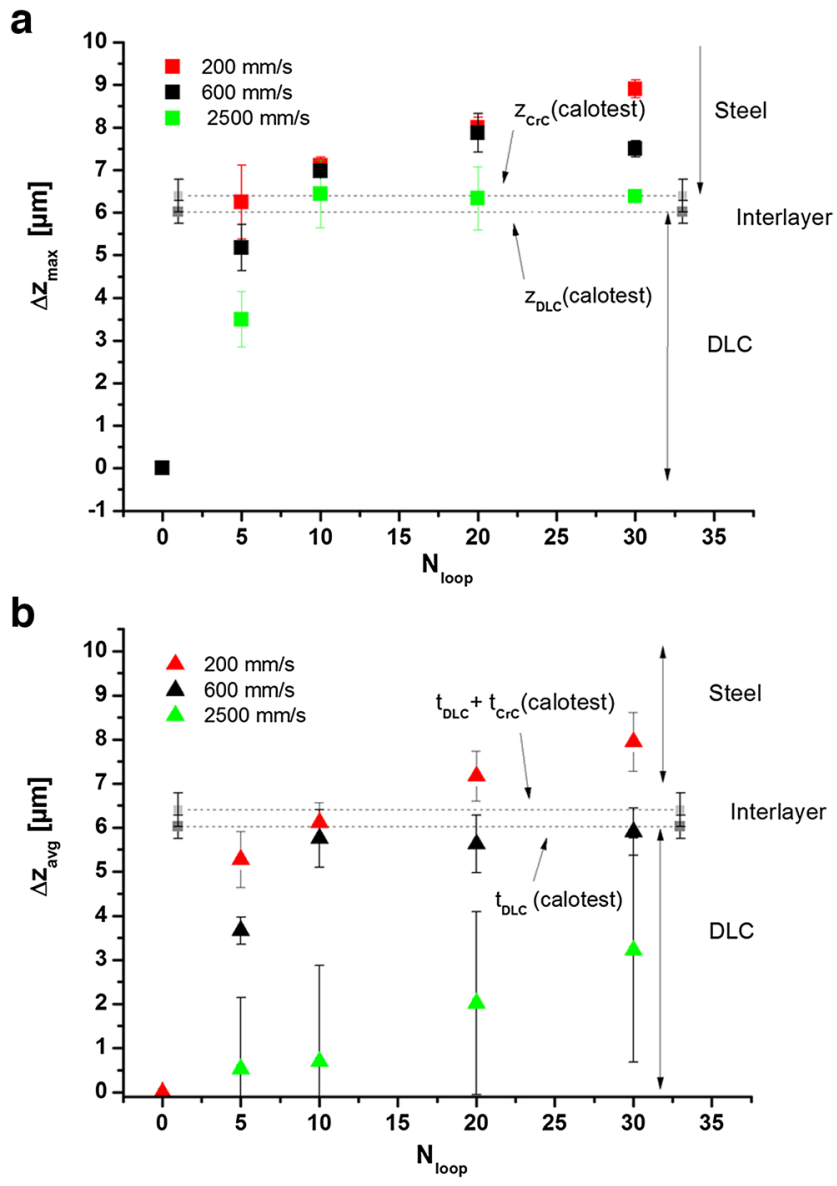

Fig. 4 Ablation depth estimators $\Delta z_{\max }$ (a) and $\Delta z_{\text {avg }}$ (b) from 3DODM profiles for different values of $N_{\text {loop }}$ and marking speed $v$. The results are compared with thickness measurement from calotest. All values are in $\mu \mathrm{m}$ homogeneous decoating textures obtained at intermediate and low lasing speed (600 and $200 \mathrm{~mm} / \mathrm{s}$, respectively). At $200 \mathrm{~mm} / \mathrm{s}$, values are almost identical using either $\Delta z_{\max }$ or $\Delta z_{\text {avg }}$ due to the planarity of the resulting texture.

A clear trend is noted for $\Delta z_{\max }$ (measuring the maximum ablation depth); as expected, $\Delta z_{\max }$ increases for increasing number of loops at all lasing speeds (Fig. 4a). However, a change in ablation rate (slope of the curves) is observed after reaching the metallic substrate $\left(z=t_{\mathrm{DLC}}\right)$, when the laser pulses are facing an increase of the ablation threshold. The average etching rate decreased from $1.1 \mu \mathrm{m} / \mathrm{loop}$ during DLC ablation to about $0.09 \mu \mathrm{m} /$ loop in the steel substrate. At $2500 \mathrm{~mm} / \mathrm{s}\left(\mathrm{OL}_{x}=70 \%\right)$, once the DLC film has been removed, the laser ablation process is occurring within the $\mathrm{CrC}$ interlayer and a saturation effect is observed for $\Delta z_{\max }$ at $N_{\text {loop }} \geq 10$ (Fig. 4b). At 600 and $200 \mathrm{~mm} / \mathrm{s}\left(\mathrm{OL}_{x}=70\right.$ and $90 \%$, respectively), the adhesion interlayer is removed after 10 passes only and the maximum ablation depth reaches the steel substrate.

On the other hand, the average depth estimator $\Delta z_{\text {avg }}$ varied stronger with marking speed (Fig. 4b). Whilst at $2500 \mathrm{~mm} /$ $\mathrm{s}$, the green markers in graph lay entirely below the DLC level $\left(t_{\mathrm{dlc}}\right)$ and the full decoating condition (Eq. 3 ) is obviously not satisfied; at $600 \mathrm{~mm} / \mathrm{s}$, only residual DLC borders are preventing the condition to be satisfied. In this case, the black markers are indeed closer to the level corresponding to the $\mathrm{DLC} / \mathrm{CrC}$ interface. At $200 \mathrm{~mm} / \mathrm{s}$, full decoating is finally verified after 10 repetitions, whereas after 5 passes, diluted residual DLC impurities on the rest surface (visible in Fig. $3 \mathrm{~g}$ ) prevent the condition to be fully satisfied.

\subsection{Effect of $N_{\text {loop }}$ and lasing speed on rest-surface roughness}

The effect of $N_{\text {loop }}$ and $v$ in air on the surface roughness $\left(R_{a}\right)$ is shown in Fig. 5a. At 2500 and $600 \mathrm{~mm} / \mathrm{s}\left(\mathrm{OL}_{x}=-25\right.$ and 


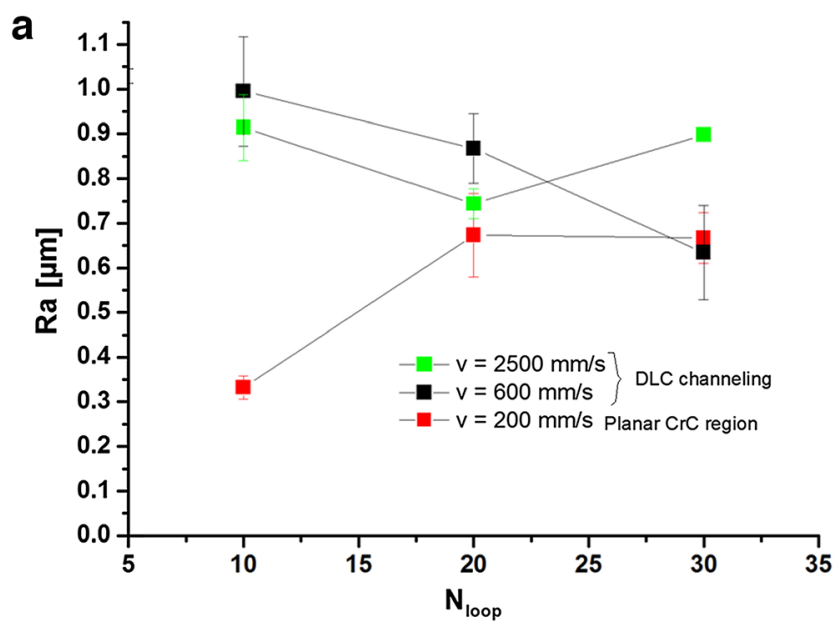

b

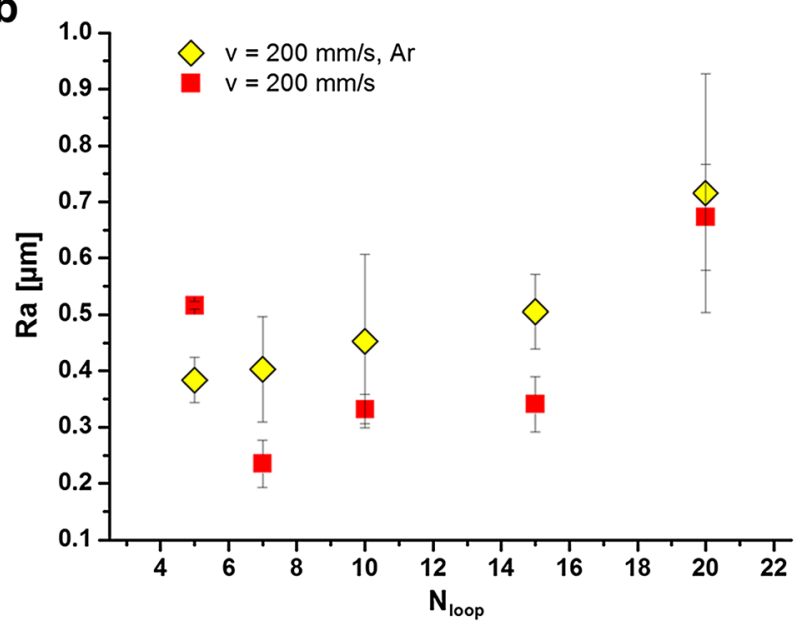

Fig. 5 a Rest surface roughness from ODM digital profilometry as a function of marking speed and number of loops. b Rest surface roughness after full decoating (with and without assist gas)

$70 \%$, respectively), high values of $R_{a}$ are reported. In the first case, this is due to absence of pulse overlap along $y$ producing alternatively ablation channels and untouched DLC zones. At $600 \mathrm{~mm} / \mathrm{s}$, a decreasing trend of $R_{a}$ for increasing $N_{\text {loop }}$ is justified by the much higher overlap percentage, resulting in a quasi decoating texture (Fig. 4c). At $200 \mathrm{~mm} / \mathrm{s}$, full decoating of the DLC film is occurring and $R_{a}$ is only determined from the metallic rest surface. Moreover, whilst reducing the number of loops from 20 to $10, R_{a}$ is decreasing of about $50 \%$. In order to confirm this trend and explore a possible minimum, further configurations were studied at $N_{\text {loop }}=5,7$ and 15. At $N_{\text {loop }}=7$, a clear minimum of $R_{a}$ was observed in air (Fig. 5b). The lowest values were reported in air $\left(R_{a} \sim 0.2-0.3 \mu \mathrm{m}\right)$ compared to $0.4-0.6 \mu \mathrm{m}$ in Ar. A different monotonic behaviour using Ar was also observed starting at $N_{\text {loop }}=5$ (yellow markers in Fig. $5 \mathrm{~b}$ ). It is finally noted that all $R_{a}$ values obtained here for DLC removal with a low power source (maximum peak power of $40 \mathrm{~W}$ and microsecond pulses) are sensibly lower compared to $R_{a}$ values obtained via high-power laser cleaning of rust and oil on a similar steel substrate (40-MW peak power, ns pulses, $\left.R_{a} \sim 2-3 \mu \mathrm{m}[9]\right)$.

\subsection{Surface oxidation and effect of $\mathrm{Ar}$}

Samples where full decoating was verified were expected to oxidise in air. Limiting oxidation is a critical requirement for subsequent redeposition and coating repair. In order to limit oxidation, the whole series at $200 \mathrm{~mm} / \mathrm{s}$ in air was repeated using $\mathrm{Ar}$ as assist gas (Ar pressure $5 \mathrm{bar}$, incidence angle $45^{\circ}$ ). EDX analysis, performed on both series - with and without $\mathrm{Ar}$ - confirmed a generally lower $\mathrm{O}_{2}$ content in Ar atmosphere. In air, different surface colours registered via optical digital microscopy (Fig. 6a, c) are clues for different oxidation mechanisms occurring on different metallic surfaces, whereas the corresponding surfaces in $\mathrm{Ar}$ appeared of the same neutral colour. Combining this information with EDX analysis on $\mathrm{Cr}$ and $\mathrm{O}_{2}$ content as a function of $N_{\text {loop }}$ (Fig. 7), the dark orange surface at $N_{\text {loop }}=7$ (Fig. 6a) was attributed to oxidation of the $\mathrm{CrC}$ interlayer. The partially blue surface observed after 15 loops (Fig. 6c) was attributed to steel oxidation of the mixture interlayer steel. It was noted that the blue content increased at higher $N_{\text {loop }}$, when the laser passes interested only the steel substrate.

The best rest-surface quality without oxidation was represented by the minimum of the $R_{a}$ curve in Ar satisfying also the decoating condition $\left(R_{a}=0.45 \mu \mathrm{m}\right.$ at $\left.N_{\text {loop }}=10\right)$. The corresponding surface (shown in Fig. 6b) appears indeed cleaner and flatter if compared to other rest surfaces in $\mathrm{Ar}$ at

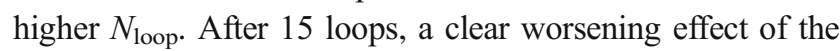
surface morphology was observed and attributed to prolonged Ar exposure (Fig. 6d). At even higher $N_{\text {loop }}(>20)$, we eventually found extended DLC debris from Ar bombardment to be redeposited at the border of the melting pool (shown in Supplementary Fig. S2).

\section{Conclusion}

In the present paper, the process of controlled laser removal of a PVD/PE-CVD DLC film has been investigated through a low-power pulsed laser source in combination with highresolution 3D digital microscopy and digital profilometry. This approach, based on the reconstruction of the postdecoating surface, allows selective removal of micrometric DLC layers with effective depth accuracy of $\pm 0.5 \mu \mathrm{m}$. Effective decoating depth and conditions for full planar decoating were studied as a function of the following lasing parameters: laser fluence, pulse overlap along orthogonal directions $x$ and $y$ and number of lasing loops. Full decoating up to the metallic interlayer of a 6- $\mu \mathrm{m}$-thick DLC film was obtained in air with the following parameter values: $F=1 \mathrm{~J} / \mathrm{cm}^{2}$, $7<N_{\text {loop }}<15$ and $v \leq 200 \mathrm{~mm} / \mathrm{s}$. The estimated DLC etching 
Fig. 6 ODM surface images after full decoating. top images Lasing in air. bottom images Lasing with $\operatorname{Ar}(5$ bar) a
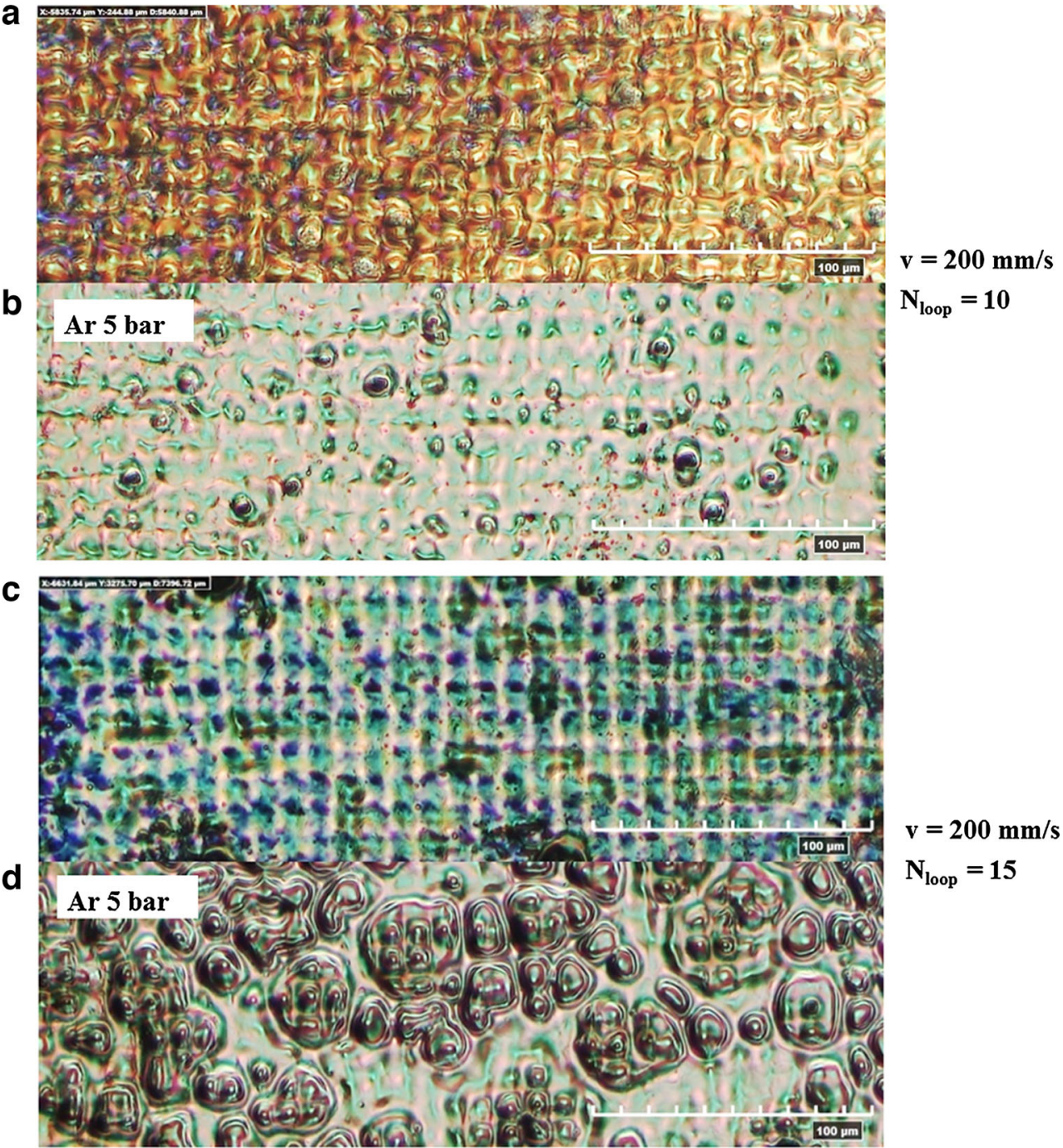

rate in this case was about $1.1 \mu \mathrm{m} /$ loop. The lowest postdecoating $R_{a}$ values $(\sim 0.2 \mu \mathrm{m})$ are reported in air with

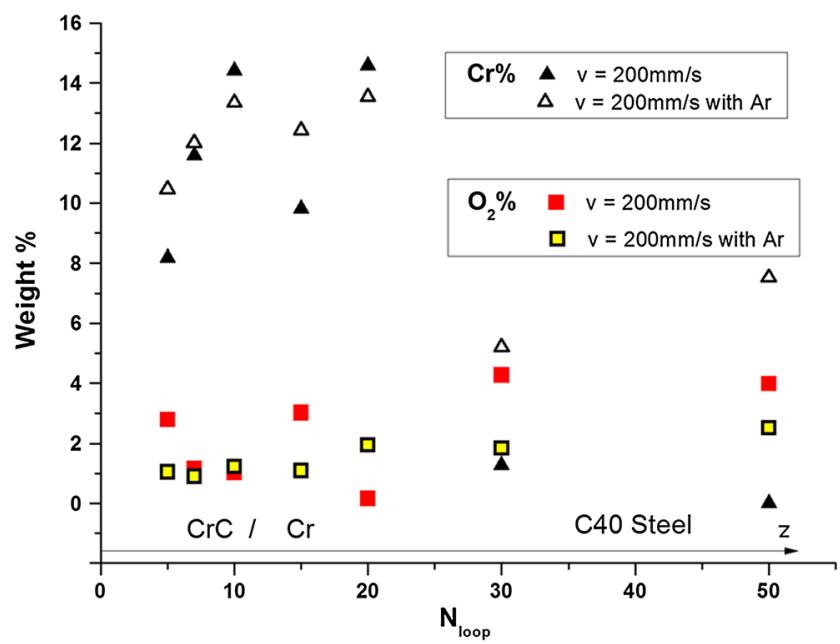

Fig. 7 The $\% \mathrm{O}_{2}$ and $\% \mathrm{Cr}$ from EDX analysis on the rest surfaces after full decoating of samples \#18-23 and \#18-23Ar oxidation of the rest surface. Limited oxidation but higher $R_{a}$ values were observed in $\mathrm{Ar}$ and attributed to prolonged Ar bombardment during the laser decoating process.

As future development of the present work, an investigation of the redeposition process of DLC films on laserdecoated surfaces (recoating) including an extensive tribomechanical characterisation of the redeposited films is planned.

Acknowledgements The authors wish to thank Protec Surface Technologies srl for providing the DLC samples. A.Z. expresses gratitude to Dr. L. Montesano, Dr. C. Pietrogalli, R. Sala and F. Faini for their experimental support.

\section{References}

1. Bewilogua K, Klaus B, Dieter H (2014) History of diamond-like carbon films - from first experiments to worldwide applications. Surf Coat Technol 242:214-225 
2. Hauert R (2008) DLC films in biomedical applications. In: C. Donnet, A. Erdemir (Eds.), Tribology of Diamond-Like Carbon Films, Springer US, pp 494-509

3. Kano M, Makoto K (2006) Super low friction of DLC applied to engine cam follower lubricated with ester-containing oil. Tribol Int 39:1682-1685

4. Kano M, Yoshida K (2010) Ultra low friction of DLC coating with lubricant. J Phys Conf Ser 258:012009

5. Thorwarth G, Falub CV, Müller U, Weisse B, Voisard C, Tobler M, Hauert R (2010) Tribological behavior of DLC-coated articulating joint implants. Acta Biomater 6:2335-2341

6. Hsiao BY (2007) Method for removing diamond-like carbon film from a substrate, https:/www.google.com/patents/US20070246371.

7. Oh J-S, Jun-Seok O, Yoshiaki K, Yuki Y, Hiroshi F, Akimitsu H (2014) Localized DLC etching by a non-thermal atmospheric-pressure helium plasma jet in ambient air. Diam Relat Mater 50:91-96. doi:10.1016/j.diamond.2014.08.009

8. Giorleo L., Ceretti E., Giardini C., Optimization of laser micromachining process for biomedical device fabrication, Int $\mathrm{J}$ Adv Manuf Technol 82 (2016) 901-907. doi: 10.1007/s00170015-7450-2.

9. Ali SN, Taha ZA, Mansour TS (2014) Laser cleaning using Qswitched Nd:YAG laser of low carbon steel alloys. Advances in Condensed Matter Physics 2014:1-6

10. Guan YC, Ng GKL, Zheng HY, Hong MH, Hong X, Zhang Z (2013) Laser surface cleaning of carbonaceous deposits on diesel engine piston. Appl Surf Sci 270:526-530

11. Konov VI (2012) Laser in micro and nanoprocessing of diamond materials. Laser Photonics Rev 6:739-766
12. Amanov A, Watabe T, Tsuboi R, Sasaki S (2013) Improvement in the tribological characteristics of Si-DLC coating by laser surface texturing under oil-lubricated point contacts at various temperatures. Surf Coat Technol 232:549-560

13. Shum PW, Zhou ZF, Li KY (2013) Investigation of the tribological properties of the different textured DLC coatings under reciprocating lubricated conditions. Tribol Int 65:259-264

14. K. Czyż, J. Marczak, R. Major, A. Mzyk, A. Rycyk, A. Sarzyński, M. Strzelec, Selected laser methods for surface structuring of biocompatible diamond-like carbon layers, Diam Relat Mater 67(2016/8) 26-40.

15. Chiu M-C, Hsieh W-P, Ho W-Y, Wang D-Y, Shieu F-S (2005) Thermal stability of Cr-doped diamond-like carbon films synthesized by cathodic arc evaporation. Thin Solid Films 476:258-263

16. Ban M, Ryoji M, Fujii S, Fujioka J (2002) Tribological characteristics of Si-containing diamond-like carbon films under oil-lubrication. Wear 253:331-338

17. Forsberg P, Gustavsson F, Renman V, Hieke A, Jacobson S (2013) Performance of DLC coatings in heated commercial engine oils. Wear 304:211-222

18. Bolelli G, Gualtieri E, Lusvarghi L, Mantini FP, Pitacco F, Valeri S, Volz H (2009 Jun 1) Thermally sprayed coatings as interlayers for DLC-based thin films. J Therm Spray Technol 18(2):231-242

19. Bolelli $\mathrm{G}$ et al (2008) Enhanced tribological properties of PECVD DLC coated thermally sprayed coatings. Surf Coat Technol 202(18):4382-4386

20. Tosti M, Pitacco F (2010) Deposition process of a nanocomposite film and apparatus EU-Patent, EP2236649 A1 